Annuaire suisse de politique de développement

$19 \mid 2000$

Pour une politique plus cohérente envers les pays en développement

\title{
La place financière suisse et la fuite de capitaux
}

Paolo Bernasconi

\section{OpenEdition}

Journals

Édition électronique

URL : http://journals.openedition.org/aspd/836

DOI : $10.4000 /$ aspd.836

ISSN : 1663-9669

Éditeur

Institut de hautes études internationales et du développement

Édition imprimée

Date de publication : 1 avril 2000

Pagination : 53-72

ISSN : 1660-5934

Référence électronique

Paolo Bernasconi, « La place financière suisse et la fuite de capitaux », Annuaire suisse de politique de développement [En ligne], 19 | 2000, mis en ligne le 18 août 2012, consulté le 07 septembre 2020. URL : http://journals.openedition.org/aspd/836 ; DOI : https://doi.org/10.4000/aspd.836 


\title{
LA PLACE FINANCIÈRE SUISSE ET LA FUITE DE CAPITAUX
}

\author{
PaOlo Bernasconi*
}

\section{INTRODUCTION: REFUGE PÉNAL ET REFUGE FISCAL'}

La masse colossale des capitaux qui continue à quitter le territoire des pays où ils ont été produits ne recherche pas seulement des occasions d'investissement plus sûres et plus rentables, mais aussi des refuges: une partie importante de ces capitaux n'ayant pas été déclarée au fisc national, il faut trouver des pays en mesure de leur garantir une immunité totale en cas d'enquêtes menées par les autorités fiscales nationales: on peut les appeler «refuges fiscaux». D'autre part, les capitaux produits illégalement, c'est-à-dire par des activités pénalement punissables, sont en quête de pays qui n'entravent ni l'accès à ces capitaux ni leur jouissance, mais par contre entravent les efforts de coopération entre les autorités pénales des différents pays: on peut les appeler "refuges pénaux". Comme toute schématisation, cette subdivision entre refuges fiscaux et refuges pénaux ne colle que partiellement à la réalité: d'une part, tous les refuges pénaux sont aussi des refuges fiscaux et, d'autre part, tous les refuges fiscaux s'efforcent de démontrer, par des mesures tantôt réelles tantôt fictives, qu'ils s'alignent sur les stratégies élaborées par la communauté internationale pour combattre la criminalité.

L'analyse de l'évolution des mesures adoptées en Suisse par les autorités et par l'économie privée permet d'établir non seulement une évaluation des Lignes directrices Nord-Sud, adoptées par le Conseil fédéral en mars 1994, concernant le transfert et le rapatriement illégaux de capitaux, mais aussi une comparaison avec les stratégies d'autres pays servant traditionnellement de refuge, dont certains, notamment le Luxembourg et le Liechtenstein, s'inspirent en grande partie du modèle suisse.

\section{LA PRÉVENTION DE L'AFFLUX DE CAPITAUX D'ORIGINE CRIMINELLE}

\section{$\square$ De l'obligation de témoigner à l'obligation de dénoncer}

Sur le plan pénal, à la punissabilité du recel (art. 160 du Code pénal suisse) et du financement du trafic de stupéfiants (art. 19 de la Loi fédérale sur les stupéfiants) sont venues s'ajouter, dès le $1^{\text {er }}$ août 1990, celle du blanchiment d'argent (art. 305bis CPS) ainsi que celle de la carence d'identification non seulement des clients d'une banque ou de tout autre intermédiaire financier ${ }^{2}$, mais aussi,

* Avocat à Lugano, professeur aux Universités de Saint-Gall et de Milan (Bocconi).

1. Abréviations utilisées dans cet article: ATF: arrêté du Tribunal fédéral; CPS: Code pénal suisse; LBA: Loi fédérale contre le blanchiment d'argent dans le secteur financier; LEIMP: Loi fédérale concernant l'entraide internationale en matière pénale. Ces termes sont définis dans l'article du même auteur paru dans l'Annuaire Suisse-Tiers Monde 1990 («Flux internationaux de capitaux d'origine illicite. La Suisse face aux nouvelles stratégies», pp. 199-229). Le présent texte constitue pratiquement la suite de cet article.

2. L'article 305ter CPS a établi des obligations identiques pour les banquiers et pour tous les autres intermédiaires financiers non bancaires. La liste de ces professionnels a été codifiée et précisée dans l'article 2 de la Loi fédérale contre le blanchiment d'argent dans le système financier, entrée en vigueur le $1^{\text {er }}$ avril 1998. 
d'après l'article 305ter CPS, des ayants droit économiques des relations bancaires ouvertes par des prête-noms ou par des sociétés de domicile. Un pas fondamental était ainsi franchi: la codification au moins indirecte, par le biais de son inscription dans le Code pénal, de l'interdiction des comptes anonymes ${ }^{3}$ a ouvert la porte aux enquêtes judiciaires permettant de repérer le produit des infractions moyennant la reconstitution des flux financiers, d'après le système du paper and asset tracing ${ }^{4}$. Cette évolution ne favorise pas seulement une procédure pénale comme telle et, donc, la prévention de la criminalité, mais aussi directement les victimes, auxquelles l'accès direct ou indirect, grâce aux enquêtes judiciaires, aux cachettes du produit des infractions commises à leur préjudice a été grandement facilités

Un progrès d'une telle envergure provient du renversement de l'attitude de l'intermédiaire financier, qui, de passif, se bornant exclusivement à réagir aux questions en cas d'investigations menées par les autorités, a dû devenir actif, communiquant spontanément aux autorités l'existence d'avoirs patrimoniaux dont on soupçonne l'origine criminelle; cette communication sera déclarée légitime grâce à l'article 305bis alinéa 2 CPS, en vigueur depuis le $1^{\text {er }}$ août 1994, puis même obligatoire, d'après l'article 9 de la Loi fédérale concernant la lutte contre le blanchiment d'argent dans le secteur financier, en vigueur depuis le $1^{\text {er }}$ avril 1998; ce dernier article prévoit en outre l'obligation du blocage interne des avoirs dénoncés par la communication. L'Office fédéral de la communication, qui fait partie de l'Office fédéral de la police, reçoit en moyenne dix communications par mois, soit une moyenne supérieure à celle que connaissent certains pays de l'OCDE, mais encore loin des milliers de communications enregistrées dans d'autres pays de l'OCDE et qui, néanmoins, n'ont pas conduit au blocage de sommes si importantes comme celles saisies en Suisse, qui ont atteint 333 millions de francs en seize mois ${ }^{6}$.

Les normes contenues dans le Code pénal et dans les lois sont parfois accompagnées d'autres normes, promulguées soit par des instances administratives, telle la Circulaire antiblanchiment de la Commission fédérale des banques, soit par

3. Le concept d' «ayant droit économique» fit son apparition dans le droit suisse grâce à la Convention de diligence de l'Association suisse des banquiers, signée le $1^{\text {er }}$ juillet 1977 par toutes les banques suisses dans le but de réagir à la profonde atteinte subie par leur image suite à l'ouverture de la procédure pénale contre les trois directeurs du Crédit Suisse de Chiasso. Par «ayant droit économique», on entend la personne physique qui exerce un pouvoir d'accès sur les avoirs déposés auprès d'un compte bancaire. Si une personne physique, pour des raisons de nature commerciale ou fiscale, ou dans le but d'essayer de cacher son identité, ouvre un compte au nom d'une autre personne physique comme prête-nom ou au nom de sociétés de domicile, elle continue à exercer son pouvoir de disposition sur les avoirs patrimoniaux grâce à un rapport juridique fiduciaire qu'elle entretient avec le prête-nom ou avec la société de domicile. A l'égard de la banque, l'ayant droit économique n'a pratiquement pas de droit, parce que tous les droits contractuels sont exercés par son fiduciaire, mais les avoirs patrimoniaux continuent à rester dans la disponibilité de l'ayant droit économique grâce au fait que son fiduciaire lui est lié par un contrat, oral ou écrit, stipulé avec l'ayant droit économique. Mais il faudra attendre le $1^{\text {er }}$ octobre 1992 pour que soit abolie la formule $B$ utilisable par les détenteurs du secret professionnel (avocats et fiduciaires) titulaires de comptes bancaires de clients, dont la Commission fédérale des banques avait en effet découvert de nombreuses utilisations abusives.

4. Toute enquête pénale ou administrative doit se fonder sur des moyens de preuve, notamment sur les preuves documentaires. Le flux de capitaux ainsi que toute transaction financière, dès qu'elle est effectuée par une banque ou par un autre intermédiaire financier, laissent une trace documentaire (paper trail) grâce à l'obligation de tenir une comptabilité. La façon la plus diffusée d'interrompre cette trace est représentée par les opérations au comptant. Pour cette raison, les législateurs de plusieurs pays depuis des années ont déclaré la guerre contre les opérations au comptant en essayant par tous les moyens de réduire de plus en plus leur diffusion. En effet, l'opération au comptant constitue une interruption de la trace documentaire et, par conséquent, un obstacle presque insurmontable pour les autorités judiciaires chargées de la reconstitution de ce flux.

5. Voir, du même auteur, «Secret et transparence pour les intermédiaires financiers »/«Geheimnis und Transparenz für Finanzintermediäre», L'expert judiciaire/Der Treuhandexperte, no 1/2000.

6. L'évolution statistique peut être consultée sur le site Internet de l'Office fédéral de la communication de Berne. 
des instances privées, telle la Convention de diligence signée par l'Association suisse des banquiers et par toutes les banques suisses. En général, ces normes complémentaires prévoient des règles de comportement plus strictes que celles prévues par les lois, dans le but notamment de sauvegarder, au sein d'un secteur particulier, un niveau de diligence plus élevé que la moyenne. Ainsi, alors que l'article 305bis du Code pénal ne punit le blanchiment d'argent que s'il est commis intentionnellement, les Directives de la Commission fédérale des banques prévoient, dans leur article 8 , que le blanchiment d'argent commis par négligence peut donner lieu à une procédure disciplinaire conduisant notamment à éloigner de l'administration ou de la direction d'une banque la personne qui s'en est rendue coupable, et cela en application de l'article 3 alinéa 2 lettre $c$ ) de la Loi fédérale sur les banques, qui, parmi les conditions de l'autorisation d'exercer l'activité bancaire, exige aussi la garantie d'une activité irréprochable de la part des responsables?.

\section{$\square$ La longue marche à travers les institutions financières}

Les premières normes concernant l'identification de l'ayant droit économique remontent à la première version de la Convention de diligence des banques (CDB), signée le $1^{\text {er }}$ juillet 1977 et révisée depuis à quatre reprises (tous les cinq ans), pour l'adapter à l'évolution qui se dessinait dans le droit pénal, dans les versions de 1992 et de 1998 des Recommandations antiblanchiment de la Commission fédérale des banques jusqu'à la codification la plus complète, celle de la Loi fédérale contre le blanchiment d'argent dans le secteur financier (LBA). Cette loi, entrée en vigueur le $1^{\mathrm{er}}$ avril 1998, a étendu les règles de conduite, jusque-là adressées aux banques, à tout le secteur des intermédiaires financiers non bancaires. La vigilance est toujours confiée aux autorités de surveillance des différents secteurs, soit la Commission fédérale des banques, l'Office fédéral des assurances et l'Office fédéral des caisses de pension, tandis que tous les autres intermédiaires, comme les gérants de fortunes, les fiduciaires, les bureaux de change, etc., sont soumis alternativement à la vigilance directe de l'Autorité fédérale de contrôle auprès du Département fédéral des finances, et aux organismes d'autorégulation (OAR) constitués par les associations professionnelles nationales ou régionales agréées par l'Autorité fédérale de contrôle antiblanchiment.

L'élargissement des règles au secteur des intermédiaires financiers non bancaires est en plein développement; il s'inspire pour une bonne part des efforts déjà entrepris par le secteur bancaire, notamment sur le plan de son organisation et de l'instruction de son personnel. Récemment, les banques ont attribué cette fonction à leur service juridique ou à un nouvel organisme, ayant pour but de vérifier que l'activité de la banque se fait dans le respect des normes de prévention du flux de capitaux d'origine criminelle (compliance office). Ces efforts, très onéreux, engendrent des coûts fixes tels qu'ils pourraient inciter, dans le secteur des intermédiaires financiers non bancaires, à une concentration des entreprises trop petites pour les supporter à elles seules.

7. A cause de l'interprétation assez large donnée par la Commission fédérale des banques à cette norme, la Commission peut communiquer à une banque si une personne, membre de son administration ou de sa direction, ne remplit plus la garantie d'activité irréprochable prévue par la loi. Par conséquent, la banque, dans le dessein de continuer à satisfaire la condition liée à son autorisation, est pratiquement contrainte à éloigner cette personne de son administration ou de sa direction. 
Pour essayer de clore le cercle, le Conseil fédéral a donné mandat à plusieurs commissions d'experts d'examiner s'il y avait lieu d'instituer, et de quelle façon, un régime légal d'autorisation et de vigilance pour tous les intermédiaires non financiers, non seulement dans le domaine des normes antiblanchiment, mais aussi dans celui de la structure, de l'organisation et des devoirs généraux de conduite, à l'instar de ce que prévoient déjà les lois fédérales sur les banques, les assurances, les fonds d'investissement, les Bourses et les commerçants de valeurs mobilières, ainsi que la législation des pays membres de l'Union européenne.

\section{$\square$ Des lacunes en commun avec les pays de l'OCDE}

L'OCDE, de par la parution, en 1990 et en 1996, de ses 40 Recommandations antiblanchiment élaborées par le Groupe d'action financière sur le blanchiment des capitaux d'origine criminelle (GAFI), continue à jouer un rôle prépondérant dans la lutte antiblanchiment, cela dans le monde entier. Cependant, tout progrès demande du temps, et l'expérience montre que le système suisse, en matière de lutte antiblanchiment, souffre de certaines lacunes - qu'il partage d'ailleurs avec les autres pays de l'OCDE:

- l'identification de l'ayant droit économique peut s'effectuer sur la base de la déclaration rendue par le client qui entre en relation d'affaires avec l'intermédiaire financier, sans que celui-ci ne soit tenu de recueillir au moins une signature de confirmation de la part de la personne désignée comme ayant droit économique ;

- l'objet du blanchiment d'argent continue à être limité aux valeurs patrimoniales, qui constituent le produit d'une catégorie limitée d'infractions pénales, soit, selon le droit suisse, les infractions qualifiées de crimes ${ }^{8}$. Cette approche a pour effet l'impunité du blanchiment d'argent du produit d'infractions qui ne rentrent pas sous la catégorie des crimes, mais sous celle des délits, entre autres le trafic illicite d'armes, le trafic de publications pédophiles, le produit de la corruption entre personnes privées, le produit de l'insider trading, etc. ;

口 l'extension très rapide des applications télématiques aux transactions commerciales et financières ( $e$-commerce et $e$-banking) risque de rendre obsolètes les pratiques de paper tracing qui ont vu le jour si lentement lors de ces dernières années. Même le GAFI en est conscient, comme le prouvent ses rapports annuels, mais la mise sur pied de contre-mesures paraît très difficile.

\section{L'ÉLARGISSEMENT DE LA COMPÉTENCE JURIDICTIONNELLE}

\section{- Punissabilité d'infractions commises en Suisse} en relation avec des infractions commises à l'étranger

L'autorité judiciaire pénale suisse est compétente pour la poursuite des infractions commises, totalement ou partiellement, sur le territoire suisse, la poursuite

8. Il s'agit des infractions punissables par une peine privative de la liberté de réclusion (art. 9 CPS). En cas d'approbation de la révision de la partie générale du CPS, qui prévoit l'abolition de la distinction entre réclusion, détention et arrêt, ces infractions correspondront alors aux infractions punissables par une peine privative de liberté supérieure à trois ans (voir art. 10 du message du Conseil fédéral no 98.038 du 21 septembre 1998). C'est pourquoi le Parlement devra veiller, lors de cette révision imminente, à ce que soit prévue une peine privative de liberté supérieure à trois ans pour les infractions les plus importantes. 
des infractions commises sur territoire étranger demeurant l'exception'. En abandonnant cette notion encore trop liée au territoire, le droit pénal suisse a prévu:

๖ la punissabilité du blanchiment, commis en territoire suisse, du produit d'un crime, même lorsque ce crime a été commis sur territoire étranger (art. 305 al. 3 CPS);

๖ la punissabilité des actes - commis en Suisse - de participation à une organisation criminelle, même lorsque celle-ci a commis ses crimes sur territoire étranger (art. 260ter CPS) ${ }^{10}$;

- le séquestre et la confiscation du produit d'infractions, même dans le cas où elles ont été commises sur territoire étranger, à condition que ce produit se trouve sur le territoire suisse (art. $59 \mathrm{CPS}$ ). Cet instrument a été renforcé grâce au renversement du fardeau de la preuve qui, dans certains cas déterminés, notamment lorsque le produit d'une infraction est en relation avec une organisation criminelle, n'est pas à la charge de l'autorité judiciaire, mais du possesseur de l'objet ou des avoirs patrimoniaux d'origine suspecte.

\section{$\square$ Poursuite des «délits mondiaux»}

La communauté internationale a considéré que certaines infractions étaient si dangereuses qu'elles devaient être poursuivies même par les pays dont le territoire n'avait pas été utilisé pour les commettre, mais sur lequel se trouvait l'auteur de l'une de ces infractions. L'infraction classique est celle du trafic de stupéfiants, mais au cours de ces dernières années la catégorie de ces «délits mondiaux » tend à s'élargir, grâce à un réseau de conventions internationales de plus en plus ample. Par conséquent, l'autorité judiciaire pénale suisse acquiert:

」 la compétence d'ouvrir"1 des procédures pénales dans le cas d'une de ces infractions, dont la punissabilité est prévue par une convention internationale, même si elle a été commise sur le territoire étranger; compétence assortie de la possibilité d'acquérir au dossier de la procédure pénale même des renseignements et des documents dont les détenteurs sont tenus par la loi à garder le secret à l'égard de tierces personnes ${ }^{12}$;

๖ la faculté d'exécuter des demandes d'entraide provenant de l'étranger concernant ces mêmes infractions;

- la compétence pour ordonner la saisie et la subséquente confiscation du produit de ces infractions ${ }^{13}$.

Dès qu'elle a la connaissance d'indices concernant l'exécution sur le territoire suisse de ces comportements punissables, l'autorité judiciaire suisse peut aussi ordonner d'office l'ouverture d'une procédure pénale pour blanchiment, d'après

9. Notamment lorsque la victime (art. 5 CPS) ou l'auteur (art. 6 CPS) de l'infraction sont de nationalité suisse.

10. La lutte contre le crime organisé, qui est généralement basé à l'étranger, a été renforcée moyennant une loi fédérale approuvée en décembre 1999 et la création d'une puissante unité spécialisée auprès de l’Office fédéral de la police.

11. Dans ce cas, d'après l'article 6bis CPS, l'autorité pénale suisse est compétente si l'accusé se trouve en Suisse.

12. Les normes concernant le secret bancaire (art. 47 de la Loi fédérale sur les banques), le secret des opérateurs de Bourse (art. 43 de la Loi fédérale sur les Bourses) et le secret d'affaires (art. 162 CPS) ne peuvent pas être opposées aux enquêtes menées par les autorités judiciaires pénales et administratives.

13. D'après l'article 64 al. 2 LEIMP, l'autorité judiciaire suisse requise d'entraide par une autorité requérante étrangère ne peut pas ordonner des mesures de restriction sur le territoire suisse qui sont assorties par une mesure coercitive (par arrestation, acquisition, saisie de biens de valeurs, convocation de témoins, libération du secret bancaire ou du secret d'affaires, etc.) si le comportement poursuivi à l'étranger n'est pas punissable d'après le droit suisse (condition de double punissabilité). 
l'article 305bis CPS, à condition qu'une infraction principale, c'est-à-dire celle dont le produit fait l'objet du blanchiment, appartienne à la catégorie des crimes, qui, dans la révision du CPS en cours d'approbation, sont définis comme les infractions punissables par une peine privative de liberté supérieure à trois ans.

\section{$\square$ La lutte contre les paiements illicites dans le commerce international}

Punissabilité de la corruption de membres d'autorités étrangères

Malgré la diffusion de la corruption des institutions publiques dans le passé lointain et proche ${ }^{14}$, ce n'est que pendant la dernière décennie que les ministères publics de nombreux pays ont finalement découvert et révélé au public l'extension de ce fléau, qui menace non seulement le fonctionnement des marchés, mais aussi celui des institutions étatiques. En conséquence, de nombreuses organisations internationales ont élaboré une série d'instruments, dont la compétence pour les autorités judiciaires d'un pays de poursuivre pour corruption même les fonctionnaires et les hommes politiques d'un autre pays ou d'une organisation internationale. Une conséquence de la ratification par la Suisse des principales conventions anticorruption ${ }^{15}$ est la révision du Code pénal suisse ${ }^{16}$, qui prévoit que le blanchiment du produit de la corruption d'un fonctionnaire étranger, soit les avantages qu'il aura perçus de la part de son corrupteur ainsi que les avantages que celui-ci aura retirés grâce à la corruption, sera désormais punissable.

Cette révision comporte aussi des conséquences dans d'autres domaines juridiques. Pour commencer, les paiements effectués par des entreprises suisses en faveur de fonctionnaires étrangers ou de leurs personnes de confiance en vue d'obtenir des avantages illicites dans le cadre d'appels d'offres, concessions, autorisations, etc., ne seront plus déductibles fiscalement ${ }^{17}$. La punissabilité des paiements en faveur d'hommes politiques et de fonctionnaires de pays étrangers ainsi que dans les organisations internationales affirme sans équivoque leur caractère illicite, ce qui rendra beaucoup plus difficile aux percepteurs de paiements corrupteurs d'obtenir, devant les autorités judiciaires civiles ou devant les juridictions arbitrales, la reconnaissance de leurs créances fondées sur les accords corruptifs.

\section{Blocage du produit de la corruption provenant de l'étranger}

Anticipant cette révision du CPS, la Commission fédérale des banques avait complété ses Directives antiblanchiment du 26 mars 1998 par l'introduction d'une série de mesures de prudence concernant les «relations d'affaires avec des personnes exerçant des fonctions politiques importantes pour un Etat étranger ou avec des personnes et sociétés qui, de manière reconnaissable, leur sont proches $\gg^{18}$.

14. Voir J.T. Noonan Jr., Bribes, Macmillan Publishing Company, New York, 1984.

15. Voir notamment la Convention de l'OCDE contre les paiements illicites dans le commerce international du $1^{\text {er }}$ décembre 1997, la Convention pénale contre la corruption du Conseil de l'Europe du 4 novembre 1998, la Convention de droit civil contre la corruption du Conseil de l'Europe du 4 novembre 1999, la Convention contre la corruption de l'Organisation des Etats américains du 29 mars 1996, etc. Pour les détails, cf. U. Cassani, La corruption de fonctionnaires: la répression pénale d'un pacte. Mélanges Schmidlin, Genève, 1999, p. 211.

16. Voir le message du 19 avril 1999 du Conseil fédéral concernant le nouvel article 322septies CPS, approuvé le 22 décembre 1999 en ratifiant la convention de l'OCDE du $1^{\text {er }}$ décembre 1997, tout en annonçant la prochaine révision de ces normes en vue de les adapter à la convention du Conseil de l'Europe du 4 novembre 1998.

17. D'après la révision des articles 27 al. 3 et 59 al. 2 de la Loi fédérale concernant les impôts fédéraux directs.

18. Voir les chiffres marginaux $\mathrm{n}^{\circ \mathrm{4}} 4,9,15,29$ et 30 de la Circulaire de la Commission fédérale des banques du 26 mars 1998 (cités dans l'annexe 5 du présent article). 
Ces mesures de prudence représentent la leçon tirée de l'expérience relative aux avoirs, mis à l'abri dans des comptes bancaires en Suisse, de dictateurs et d'hommes politiques. Dans le cadre de cette stratégie, le Conseil fédéral a ordonné, le 17 mai 1999, le blocage des comptes et des avoirs patrimoniaux du président du Zaïre, Mobutu. En outre, le recours lancé par ce dernier suite au blocage de 4,8 millions de francs fut rejeté par le Tribunal fédéral. Une mesure de blocage analogue des avoirs patrimoniaux du président yougoslave Milosevic et de ses plus proches collaborateurs politiques fut aussi ordonnée par le Conseil fédéral. Auparavant, en 1998, les autorités judiciaires pénales avaient saisi environ 20 millions de francs en exécution d'une demande d'entraide concernant l'ancien premier ministre pakistanais Benazir Bhutto, ainsi qu'environ 3,5 millions de francs de l'ancien président de la république du Mali, Moussa Traoré; en 1999, 650 millions de dollars furent saisis auprès de trois banques de Zurich et de sept banques de Genève, en relation avec le clan de Sani Abacha, ancien président du Nigeria, et cela déjà avant la présentation de la demande d'entraide dans l'intérêt de la procédure pénale contre 14 personnalités de ce régime pour la soustraction de biens de l'Etat. De son côté, le Tribunal fédéral rejeta le recours contre la remise aux autorités philippines de 120 millions de francs, qui avaient été saisis en 1986 auprès de comptes bancaires ouverts au nom Ferdinand Marcos et de ses proches. En outre, des centaines de commissions rogatoires étrangères furent exécutées en Suisse dans l'intérêt de procédures judiciaires menées pour corruption contre des hommes politiques et des fonctionnaires, notamment dans le cadre de l'opération Mani Pulite lancée par des procureurs publics en Italie, ainsi que dans l'intérêt des procédures pénales concernant la société française Elf Aquitaine, la société espagnole Matesa et beaucoup d'autres, du Canada au Mexique, de la Corée à l'Inde, de l'Ukraine à la Fédération russe; elles aboutirent à un changement important de la jurisprudence de la part du Tribunal fédéral, qui a autorisé la coopération suisse aussi dans l'intérêt des procédures menées à l'étranger pour violation de la législation étrangère concernant le financement public des partis politiques ${ }^{19}$. Une demande d'entraide vient d'être exécutée qu'en voici une nouvelle: l'autorité judiciaire pénale allemande a déjà demandé l'assistance des autorités judiciaires pénales suisses dans le but de découvrir les cachettes des millions de francs suisses que le parti démocrate-chrétien allemand, contrairement à ce qu'impose la législation allemande, n'avait pas enregistrés dans sa comptabilité.

En effet, on peut constater que malgré l'effort de sensibilisation du secteur financier privé mené par le législateur ainsi que par les autorités judiciaires pénales et par la Commission fédérale des banques, certaines mesures de prévention n'ont pas encore abouti à un résultat envisagé. L'exemple des avoirs de Mobutu et de sa famille est malheureusement très éloquent: en effet depuis le blocage, en 1986, des avoirs de Marcos et de sa famille, le président Mobutu avait souvent été évoqué dans la presse comme futur «candidat» à un blocage. Cependant, on a dû constater que, après la chute de son régime, certaines banques suisses avaient continué à abriter et gérer une partie de son patrimoine.

19. Voir le jugement du Tribunal fédéral 124 II 184. 


\section{LE RENFORCEMENT DE LA COOPÉRATION INTERNATIONALE ENTRE LES AUTORITÉS}

\section{$\square$ De l'«entrave» à l'entraide internationale en matière pénale}

Pendant des décennies, la coopération internationale entre les autorités judiciaires pénales des différents pays s'est heurtée à l'indifférence et à la méfiance. La ratification de nombreux instruments internationaux ${ }^{20}$ a marqué un progrès colossal, dont le mérite revient aussi à certaines autorités dynamiques, à quelques rares enquêteurs et au Tribunal fédéral, qui a établi le principe de l'utilité potentielle des moyens de preuve. Selon ce principe, tous les moyens de preuve dont on ne peut pas exclure une utilité pour l'enquête étrangère doivent être transmis à l'autorité judiciaire étrangère. On peut aussi en déduire que, en cas de doute concernant l'utilité d'un moyen de preuve - y compris un document bancaire -, l'autorité judiciaire suisse requise doit remettre ce moyen de preuve à l'autorité judiciaire étrangère requérante. Pour cette raison, on peut conclure qu'aujourd'hui le système suisse de l'entraide est régi par le principe in dubio pro rogatoria ${ }^{21}$. L'autorité judiciaire suisse requise est même habilitée à transmettre des moyens de preuve qui n'avaient pas été demandés par l'autorité judiciaire requérante, généralement parce qu'elle n'en avait pas encore connaissance. En outre, l'autorité judiciaire suisse requise peut même transmettre des renseignements spontanément à l'autorité judiciaire étrangère concernant des infractions qui ont été commises sur le territoire étranger, dont l'autorité suisse a eu connaissance dans le cadre de ses enquêtes ${ }^{22}$. Mis à part un système de recours facilitant les manœuvres retardatrices, en matière d'entraide internationale, la doctrine et la jurisprudence suisses demeurent un modèle pour presque tous les pays du monde, y compris ceux de l'Union européenne, lesquels, malgré leur appartenance à une organisation qui a accompli l'unification des marchés et de la monnaie, n'ont pas encore été à même de réaliser un système commun de coopération en matière pénale. La preuve en est que le système vétuste des commissions rogatoires trouvera sa confirmation l'année prochaine, grâce à une convention d'entraide ratifiée par les pays membres de l'Union européenne. Il s'agit d'un texte qui ne fait que reprendre des techniques et des pratiques ${ }^{23}$ bien connues, de sorte que sa ratification ne poserait aucun problème à la Suisse ${ }^{24}$.

\section{$\square$ De l'entraide en matière pénale à l'entraide en matière administrative}

Grâce à la révision de la Loi fédérale sur les banques (art. 23sexies) ainsi que de la nouvelle loi sur les Bourses et les commerçants de valeurs mobilières (art. 38), la Commission fédérale des banques est désormais autorisée, depuis le

20. A la ratification des conventions du Conseil de l'Europe concernant l'extradition des personnes de 1957, l'extradition de moyens de preuve de 1959, la confiscation ou l'extradition du produit du crime du 10 octobre 1990 s'est ajoutée la ratification de traités bilatéraux d'entraide avec les quatre pays limitrophes de la Suisse ainsi qu'avec l'Australie, le Canada et les Etats-Unis, complétés par des traités pour la coopération en matière policière, notamment avec l'Allemagne, l'Autriche, la France et le Liechtenstein.

21. D'après le principe de faveur (Günstigkeitsprinzip) établi par l'ATF 122 II 367; cf. aussi R. Zimmermann, L'entraide judiciaire internationale en matière pénale, Berne, 1999, p. 368

22. Ce mécanisme a été prévu par l'article 10 de la Convention n ${ }^{\circ} 141$ de Strasbourg contre le blanchiment du 8 octobre 1990, ainsi que par l'article 67a LEIMP.

23. Ainsi notamment la solution choisie pour la surveillance des télécommunications parât très soucieuse du principe de la souveraineté territoriale.

24. Au surplus, la Suisse y trouverait même de l'intérêt car le projet de cette Convention prévoit l'élargissement du principe de la spécialité - un pilier traditionnel de la pratique suisse -, même dans le cas de transmission spontanée de moyens de preuve à l'étranger. 
$1^{\text {er }}$ février 1997, à échanger des renseignements et des moyens de preuve avec ses homologues étrangers, non seulement dans le but de protéger le fonctionnement des marchés, mais aussi dans le cadre d'enquêtes administratives ouvertes sur des suspects d'insider trading ou de manipulation de cours de titres cotés en Bourse. Depuis presque deux années, une centaine de demandes de coopération ont été soumises à la Commission fédérale des banques, qui en a accepté la grande majorité. D'après la jurisprudence du Tribunal fédéral, il suffit qu'il y ait le soupçon d'une opération destinée à l'insider trading ${ }^{25}$ pour que l'autorité suisse transmette les moyens de preuve demandés à l'autorité administrative étrangère, tout en sachant que celle-ci, en règle générale, est tenue, d'après son droit national, à transmettre le résultat de la coopération internationale à l'autorité judiciaire pénale, dont les dossiers sont accessibles aussi à l'autorité fiscale. La transmission de la part de la Suisse ne se fait que sous la condition - d'après le principe de la spécialité ${ }^{26}$, bien rodé dans le cadre de l'entraide en matière pénale - de ne pas utiliser ces moyens de preuve à des fins fiscales.

Même les autorités douanières ont été autorisées à échanger des informations et des moyens de preuve avec leurs homologues étrangères, dans le dessein de donner, au moins partiellement, satisfaction au nombre croissant de doléances adressées à la Suisse à cause de l'hospitalité accordée à des activités qui violent systématiquement les règles douanières étrangères, notamment celles des pays membres de l'Union européenne ${ }^{27}$.

\section{LA COOPÉRATION INTERNATIONALE EN MATIÈRE FISCALE}

\section{$\square$ Une grande exception qui dépasse le principe}

La Loi fédérale sur l'entraide internationale en matière pénale, en vigueur depuis le $1^{\text {er }}$ janvier 1983, a codifié dans son article 3 la doctrine sans cesse réitérée par le Parlement ainsi que par le gouvernement suisses: pas de coopération avec l'étranger en matière fiscale, sauf dans l'intérêt d'une procédure fiscale menée à l'étranger pour le chef d'escroquerie en matière fiscale, c'està-dire, notamment, d'épargne fiscale illégale obtenue moyennant l'utilisation de faux documents, tels que bilans, comptes de pertes et profits, factures, contrats, etc., par des milliers d'entreprises étrangères; cette norme aurait pu donner lieu à des milliers de demandes d'entraide. En réalité, depuis l'entrée en vigueur de cette norme qui autorise l'entraide dans l'intérêt des procédures étrangères pour escroquerie en matière fiscale, elle n'a été utilisée que vingt à trente fois par année par les autorités étrangères, principalement celles des pays scandinaves, de l'Allemagne et de la Grande-Bretagne, les pays méditerranéens et extra-européens demeurant presque totalement absents.

25. En général, les autorités de vigilance sur la Bourse considèrent suspecte une opération d'achat ou de vente de titres effectuée dans une période suivant de près un fait qui influence d'une manière importante le cours de ces titres, comme par exemple l'acquisition de la société cotée en Bourse par une autre société ou une affiliation avec une autre société.

26. D'après ce principe, les moyens de preuve transmis par l'autorité judiciaire d'un pays à l'autorité judiciaire d'un autre pays dans le cadre de l'entraide internationale en matière pénale ne peuvent être exploités par l'autorité requérante que dans le but pour lequel l'entraide avait été demandée et octroyée. Ce principe, que la Suisse ne cesse d'infirmer à chaque ratification d'une convention internationale, est destiné notamment à empêcher que l'autorité judiciaire étrangère n'utilise les moyens de preuve, remis par l'autorité suisse dans le cadre de l'entraide, pour des cas de délits fiscaux.

27. Par conséquent, la Suisse a signé le 9 juin 1997 un protocole additionnel à l'Accord du 22 juillet 1972 entre la Suisse et la CEE. 


\section{$\square$ Prochaines étapes: l'extradition des personnes}

et des avoirs patrimoniaux en relation avec la fraude fiscale

L'exception vue ci-dessus, soit la coopération des autorités suisses dans l'intérêt de procédures menées à l'étranger pour le chef d'escroquerie en matière fiscale, ne vaut que pour l'échange de renseignements et de moyens de preuve. Ainsi, la Suisse ne concède pas l'extradition de personnes recherchées par les autorités étrangères pour escroquerie en matière físcale, le Protocole additionnel ${ }^{\circ} 99$ de la Convention européenne sur l'entraide internationale en matière pénale ayant été rejeté par le Parlement. Cependant, suite à la ratification de ce protocole par le Luxembourg, il n'est pas exclu que le Parlement suisse revienne sur sa décision.

Il en va de même pour la remise du produit de l'escroquerie en matière fiscale, qui n'est pas admise par l'autorité suisse car elle équivaudrait à une coopération suisse au recouvrement d'une créance fiscale étrangère. Sur cette question la doctrine est unanime, mais l'Office fédéral de la police, dans ses Directives concernant l'entraide internationale en matière pénale promulguées en 1998 (chapitre 2.2, p. 11), a exprimé un avis contraire. Si cet avis devait susciter un écho favorable de la part du Tribunal fédéral, il resterait encore à préciser la nature et l'étendue des avoirs patrimoniaux soumis à la saisie en vue de leur remise à l'Etat étranger ou de leur confiscation: s'agirait-il d'une valeur équivalente à l'épargne fiscale obtenue grâce aux manœuvres frauduleuses, telle notamment l'utilisation de faux documents, ou s'agirait-il de tous les avoirs n'ayant pas été déclarés au fisc?

\section{$\square$ Réticence politique, dynamisme judiciaire}

La coopération internationale entre autorités fiscales marque le pas, non seulement entre la Suisse et les autres pays, mais aussi parmi les pays de l'OCDE et même parmi ceux de l'Union européenne. La preuve en est que la Convention pour l'entraide internationale mutuelle entre autorités fiscales, approuvée en 1988 par l'OCDE et par le Conseil de l'Europe après une longue marche institutionnelle, n'a été ratifiée à ce jour que par six pays. Rien d'étonnant si la Suisse et le Luxembourg ne ratifieront pas cette convention tant qu'elle ne l'aura pas été par les pays à fiscalité forte.

Même chez les autorités fiscales des pays membres de l'Union européenne l'application et la mise en œuvre des premières directives en la matière ne donnent pas satisfaction. C'est une fois de plus aux autorités judiciaires pénales de prendre l'initiative, notamment en Allemagne et en Belgique: en 1999, des initiatives extraterritoriales ont été adoptées par les autorités judiciaires de Koblenz, qui ont condamné deux hauts dirigeants d'une banque allemande pour participation aux infractions fiscales commises par leurs clients, ces deux personnes ayant aidé leurs clients à transférer, du territoire allemand auprès des filiales de cette même banque basées au Luxembourg, leurs avoirs non déclarés au fisc allemand. De même, le dirigeant d'une banque luxembourgeoise a été condamné par le Tribunal de Bruxelles à cause d'un comportement similaire. De telles initiatives judiciaires pourraient à la longue exercer un résultat beaucoup plus percutant sur la Suisse que les pressions exercées à l'encontre du secret bancaire suisse par quelques organisations internationales ${ }^{28}$.

28. Voir notamment le rapport approuvé en avril 1998 par l'OCDE avec l'abstention du Luxembourg et de la Suisse concernant la concurrence fiscale dommageable et le secret bancaire. 


\section{$\square$ Le droit pénal: cheval de Troie pour le droit fiscal}

La lutte contre le crime organisé ainsi que contre l'un de ses instruments préférés, le blanchiment d'argent, continue à jouer un rôle prépondérant dans le renforcement et l'amélioration des instruments de répression pénale. Les organisations internationales ne cessent d'approuver des textes qui prônent cette tendance $^{29}$, et ce malgré que la criminalité organisée continue son expansion, en raison de phénomènes politiques tels que l'effondrement des institutions étatiques dans les pays en transition de l'Europe de l'Est ou encore en raison de l'inefficacité des instruments de répression et de prévention. Face à la demande pressante de l'opinion publique, alimentée par la peur et l'insécurité, les autorités politiques des pays occidentaux, sous la pression des pays anglo-saxons, qui ne font pas de distinction entre infractions de droit pénal et infractions de droit fiscal, paraissent sur le point de lier la répression des infractions pénales à la répression des infractions fiscales: au sein du Groupe d'action financière sur le blanchiment d'argent de capitaux (GAFI), il est depuis longtemps question de convaincre les Etats membres d'insérer l'escroquerie en matière fiscale ou la fraude fiscale dans les infractions principales en amont du blanchiment d'argent. Cette révision du droit pénal matériel aurait pour effet l'ouverture de procédures pénales par les autorités judiciaires d'un pays contre les personnes ayant mis à l'abri sur le territoire de ce pays le produit de l'escroquerie et de la fraude commises au préjudice du fisc national ou du fisc étranger. La même révision pourrait avoir lieu concernant la participation à une organisation criminelle, participation qui, d'après l'article 260ter CPS, n'est punissable que si l'organisation commet des activités qualifiées de crimes: il n'est en effet pas exclu que les pays membres des organisations internationales acceptent d'étendre la punissabilité de la participation à une organisation criminelle aux cas où les activités de cette dernière relèvent de l'escroquerie ou de la fraude fiscales. L'Union européenne, très préoccupée par l'ampleur colossale des fraudes commises au préjudice de ses intérêts financiers, joue un rôle moteur en faveur de ces révisions. C'est pourquoi elle tente d'insérer la fraude contre ses intérêts financiers parmi la catégorie de crimes les plus graves, dans le but de favoriser la coopération internationale entre les autorités judiciaires en matière pénale ainsi que la saisie et la confiscation du produit de ces fraudes et la punissabilité du blanchiment de ce produit.

\section{$\square$ Le territoire suisse: paradis fiscal pour les sociétés off shore}

- Les sociétés de domicile constituées dans les pays off shore sont toujours utilisées dans le cadre de la criminalité économique et du blanchiment du produit de trafics illicites, mais cela ne concerne qu'une partie minime d'entre elles, la majorité étant constituée et utilisée afin de dissimuler des avoirs patrimoniaux qui n'ont pas été déclarés au fisc national suisse, ou étranger. Au nom de ces sociétés off shore, des centaines de milliers de relations bancaires ont été ouvertes auprès de banques et de sociétés financières en Suisse et ailleurs. Depuis quelques années, pour satisfaire aux exigences de la clientèle étrangère préoccupée par les nouvelles médiatiques au sujet de la coopération internationale en matière pénale, les banques en Suisse mettent à disposition leurs filiales constituées dans des pays off shore, dont

29. Pro multis voir les plans contre le crime organisé de la CEE. 
notamment les îles Anglo-Normandes, Monaco, le Luxembourg, les Bahamas, les îles Cayman et les îles Vierges britanniques. En réalité, la gestion de ces patrimoines continue à se faire auprès de la banque en Suisse, ce qui permet aux autorités judiciaires ainsi qu'à la Commission fédérale des banques d'obtenir des renseignements et des moyens de preuve également sur la relation bancaire off shore ouverte au nom de laquelle ont été déposés les avoirs patrimoniaux gérés en Suisse. Cet accès a été autorisé par le Tribunal fédéral ${ }^{30}$, même dans le but de retransmettre le résultat de l'enquête aux autorités étrangères dans l'intérêt de leurs investigations sur des suspects d'insider trading.

Cependant, la relation d'affaires avec une banque off shore continue dans l'ensemble à jouir d'une grande fortune auprès des contribuables suisses et étrangers, ce qui explique la présence, dans les îles Anglo-Normandes, aux Bahamas et aux îles Cayman, de nombreuses filiales de banques qui sont aussi autorisées à exercer leur activité sur le territoire suisse. En général, ce phénomène ne constitue pas une entrave aux enquêtes des autorités judiciaires pénales: en effet, d'une part, les Directives antiblanchiment de la Commission fédérale des banques (art. 2) interdisent aux banques autorisées en Suisse l'utilisation de filiales off shore dans le dessein d'esquiver les Directives elles-mêmes; d'autre part, l'autorité judiciaire pénale suisse peut interroger comme témoin l'employé de banque suisse et obtenir des renseignements sur les relations bancaires ouvertes auprès de filiales off shore. La seule entrave consiste dans l'impossibilité pour l'autorité judiciaire pénale suisse de saisir, voire de confisquer, les avoirs déposés auprès d'une filiale off shore, cela ne pouvant s'accomplir que par le biais exclusif d'une demande d'entraide envoyée aux autorités du pays off shore. Dans la réalité toutefois, un magistrat suisse a déjà trouvé un stratagème pour contourner cette entrave: il s'agit de notifier à la banque suisse que les valeurs déposées auprès de sa filiale étrangère sont d'origine illicite, de sorte que ses employés encourent le risque d'ouverture d'une procédure pénale en Suisse pour blanchiment d'argent dans le cas où, suivant les instructions de leurs clients, ils seraient amenés à transférer ailleurs ces biens déposés auprès de la filiale off shore. Ce stratagème paraît actuellement inconnu des autorités judiciaires étrangères, pour lesquelles les pays off shore continuent à représenter une zone interdite et inaccessible à la coopération internationale en matière pénale.

- En matière fiscale continue de subsister le principe - pour paraphraser l'adage - Punissabilité au-delà des Pyrénées, impunité au-deçà. Par conséquent, de très nombreuses sociétés fiduciaires basées en Suisse continuent de mettre sur pied des mécanismes sociétaires permettant aux entreprises contribuables à l'étranger de transférer sur des comptes bancaires ouverts au nom des actionnaires de ces entreprises la différence résultant de procédures de sous- et surfacturation, et cela en toute impunité, puisque ces comportements ne sont pas punissables d'après le droit suisse, mais seulement d'après le droit fiscal étranger. Cette «fabrique» de factures totalement ou partiellement fausses ne court qu'un risque moindre d'une procédure suisse pour faux dans les titres à cause de l'incertitude constante de la jurisprudence du 
Tribunal fédéral dans l'application de l'article 251 CPS punissant le faux dans les titres. Néanmoins, ces fiduciaires s'exposent:

- au risque d'une procédure pénale étrangère qui peut aboutir à leur arrestation dans le cas où elles devraient séjourner sur le territoire de l'Etat intéressé ;

- au risque de l'exécution de la part des autorités suisses d'une demande étrangère d'entraide pour la saisie de documents et pour l'interrogatoire en qualité de témoins ou de personnes accusées. En effet, ces systèmes de sous- et surfacturation sont très souvent qualifiés par l'autorité suisse de fraude fiscale, ce qui donne lieu à l'acceptation des demandes d'entraide étrangères; cependant, l'exécution de ces demandes exigeant plusieurs mois, les contribuables étrangers ont largement le temps de prendre toute disposition nécessaire à la protection de leurs intérêts patrimoniaux.

C'est pourquoi, malgré ces risques, on peut considérer que le territoire suisse continue de représenter une région optimale pour des activités, des transactions et des sociétés cherchant à commettre des fraudes au préjudice du fisc étranger, non seulement par le biais de sociétés suisses dotées d'un régime fiscal privilégié, mais aussi grâce à une masse colossale de sociétés étrangères ayant leur siège à Londres ou dans les pays off shore les plus renommés pour la qualité de leurs services, tels les îles Anglo-Normandes, le Luxembourg, le Liechtenstein, le Panama, les îles Cayman, les Bahamas, les Antilles néerlandaises et les îles Vierges britanniques ${ }^{31}$.

- Dans le collimateur des organisations internationales et notamment du Comité fiscal de l'OCDE, qui ont déclaré la guerre contre la concurrence fiscale déloyale, se trouvent les régimes fiscaux privilégiés accordés aux sociétés de domicile et aux sociétés auxiliaires qui ne servent que de support aux activités menées hors du territoire des paradis fiscaux. Raison pour laquelle aussi le nom de la Suisse a été mentionné dans la liste des paradis fiscaux par la Legge finanziaria 1992 de la République italienne, qui ne reconnaît plus la déduction des dépenses facturées par des sociétés résidant dans ces pays. Il s'agit de mesures législatives qui se répandent de plus en plus dans les pays à fiscalité forte: ayant constaté que les entreprises soumises à la souveraineté fiscale des pays à fiscalité forte essaient d'augmenter leurs coûts moyennant des factures totalement ou partiellement fictives émises par les sociétés ayant leur siège dans des pays off shore, les pays à fiscalité forte n'autorisent plus la déduction des revenus imposables des coûts qui n'ont comme justification que des factures émises par une société ayant son siège dans un pays off shore. C'est encore une fois l'Italie qui a pris l'initiative de mentionner la Suisse dans la liste des paradis fiscaux, dans son annexe à la loi du 4 mai 1999, qui a introduit le renversement du fardeau de la preuve à la charge des citoyens italiens déclarant avoir pris domicile en Suisse ou dans l'un de 59 autres paradis fiscaux. En effet, en Suisse comme dans beaucoup d'autres pays à la chasse de riches contribuables et de riches successions, tels que la Principauté de Monaco, Andorre, Malte et Chypre en Europe, et des pays qui offrent un deuxième passeport pour quelques sous, il

31. Selon le rapport d'activité annuel de 1998 de l'Office de la communication de l'Office fédéral de la police, $12 \%$ des communications concernent des fonds déposés en Suisse par des sociétés ayant leur siège aux îles Vierges britanniques. 
existe un rapport inversement proportionnel entre l'importance de la fortune du contribuable étranger et l'intensité des vérifications concernant la réalité de son domicile sur territoire suisse: plus ce contribuable sera fortuné, moins rigoureuses les conditions pour la concession du domicile et moins rigoureux encore les contrôles pour vérifier s'il ne s'agit pas d'un domicile fictif.

La doctrine du new public management étant interprétée par les pouvoirs publics eux-mêmes comme la maximisation du profit à tout prix, les cantons suisses se livrent à une concurrence fiscale toujours plus acharnée. Nul ne s'étonnera, par conséquent, si, en dépit des déclarations des organisations internationales, cette concurrence continuera de trouver des alliés importants même en Suisse.

\section{EN GUISE DE CONCLUSION.}

\section{LE COMBAT CONTRE LES SOCIÉTÉS OFF SHORE: UN DÉFI POLITIQUE}

Les responsables de la stratégie de la lutte contre le crime organisé ont constaté, pendant des décennies, que les sociétés de domicile situées dans les pays off shore ont été - et sont toujours - considérées comme l'instrument préféré du crime organisé, du terrorisme et du blanchiment d'argent. Leurs propositions d'intervention se sont toujours heurtées au principe de la souveraineté des Etats. Cet obstacle commence à s'effriter pour les raisons suivantes :

- La législation internationale et nationale destinée à assurer le bon ordre et le fonctionnement des marchés constitue un réseau de normes précises et étendues mondialement. Il suffit de songer aux normes contre la concurrence déloyale, contre les trusts, contre la concentration dans le secteur des médias, pour la vigilance sur les marchés bancaires et boursiers, etc. Les autorités de surveillance internationales et nationales ont constaté que leur activité se heurtait à l'utilisation diffuse de sociétés de domicile ayant leur siège dans des pays off shore.

- Toute politique économique commune sur le plan fiscal, notamment à l'intérieur des communautés internationales de plus en plus intégrées sur le plan politique et économique, dont l'Union politique européenne est l'exemple le plus extrême, se heurte aux violations et aux abus rendus possibles grâce à l'utilisation de sociétés de domicile ayant leur siège dans des pays off shore. Un instrument typique de la stratégie qui s'en est suivie est représenté par le Code de conduite (Ecofin), signé le $1^{\text {er }}$ décembre 1997 par les pays membres de l'Union européenne, qui prévoit dans une première phase le gel et, dans une deuxième phase, le démantèlement de tous les privilèges de nature físcale concédés aux entreprises non résidantes. La force de ce Code de conduite - et la difficulté de sa mise en œuvre - réside dans son article qui prévoit l'engagement, de la part des pays membres de l'Union européenne, à faire appliquer le contenu de ce Code également sur le territoire des pays dépendants, d'une façon ou d'une autre, ce qui touche notamment les îles Anglo-Normandes, Gibraltar, Madère, les Antilles néerlandaises, etc.

D'autres propositions de mesures concernant les pays off shore surgissent comme des champignons, d'une façon désordonnée, sous les formes les plus diverses, dans le cadre des organisations internationales, engendrant un 
marasme d'initiatives qui fait suite à un sommeil prolongé pendant des décennies. Afin que ces stratégies ne restent que des «tigres de papier», il faut éviter de diaboliser les pays off shore comme tels et chercher plutôt à identifier avec précision les mécanismes qui facilitent les abus, en précisant que les instruments préférés de ces mécanismes sont les sociétés de domicile, c'est-à-dire des personnes morales qui n'exercent aucune activité industrielle ou commerciale et ne possèdent ni personnel ni locaux, ne disposant en général que d'une simple adresse, d'où leur définition de "sociétés boîte aux lettres".

La globalisation du marché est en cours et, avec elle, la globalisation de l'injustice, de la pauvreté et de la guerre. Si l'on veut réaliser la globalisation des normes et, pour y parvenir, la globalisation de la transparence et de la vigilance, il est indispensable aussi de s'attacher à la lutte contre les moyens abusifs les plus répandus et les plus efficaces, dont notamment les sociétés de domicile ayant leur siège dans des pays off shore. Cette lutte représente un défi non seulement pour les autorités politiques de la Suisse, mais aussi pour les autorités politiques des autres pays et pour les organisations internationales. 


\section{Annexe 1}

\section{Evolution des normes suisses contre l'afflux de capitaux d'origine illicite}

1977 Obligation d'identification de l'ayant droit économique (ADE) d'après la Convention de diligence des banques suisses

1981 Entraide internationale pour escroquerie en matière fiscale (EIMP).

1988 Punissabilité de l'insider trading (art. 161 CPS).

1990 Punissabilité

a) du blanchiment d'argent (art. 305bis CPS);

b) de la négligence dans l'identification de l'ADE (art. 305ter CPS).

Ratification de la Convention de Strasbourg no 141 concernant le dépistage, la saisie, la confiscation et le blanchiment d'argent d'origine illicite.

1992 Enregistrement centralisé de l'ADE (introduit par la Circulaire antiblanchiment de la Commission fédérale des banques).

1994 a) Droit de communication (art. 305ter al. 2 CPS).

b) Punissabilité de l'association criminelle (art. 260ter CPS).

c) Renversement du fardeau de la preuve pour la confiscation d'avoirs appartenant au crime organisé (art. 59 chiffre 3 CPS).

1996 Obligation de témoigner pour les avocats étendue aux renseignements acquis hors de l'exercice de leur activité juridique au sens strict (ATF 120 lb 112; 117 la 341; Semaine judiciaire 1996, 453).

1997 Transmission à l'étranger des moyens de preuve même en cas d'utilité potentielle (ATF 122 ॥ 367).

1998 Obligation de communiquer (art. 9 LBA) les transactions concernant des fonds dont on soupçonne l'origine criminelle et de les bloquer.

1999 a) Enregistrement centralisé des ayants droit de signature (introduit par la Circulaire 1999 de l'Association suisse des banquiers).

b) Transmission à l'étranger de renseignements concernant les clients de filiales off shore (ATF $125 \|$ 400).

2000 Punissabilité de la corruption d'autorités étrangères (art. 322ter CPS), en application des conventions de l'OCDE et du Conseil de l'Europe contre la corruption. 


\section{ANNEXE 2 \\ Les défis futurs dans la lutte contre l'afflux de capitaux d'origine illicite}

\section{Préambule}

Cette annexe dresse la liste des mesures examinées dans le cadre de différentes organisations internationales ainsi que d'organisations non gouvernementales. II s'agit de mesures qui touchent le système juridique de nombreux pays, indépendamment de leur appartenance ou non à l'OCDE et de leur position en tant que pays à fiscalité forte ou pays off shore. L'étude des arguments de nature politique, économique et juridique pour ou contre l'une ou l'autre de ces mesures dépasse le cadre de cet article, dont l'objectif est de mettre à disposition des critères utiles pour l'examen de la liste de ces mesures.

\section{Première catégorie: mesures contre les infractions de droit commun}

1. Elargissement de la catégorie des infractions principales en amont du blanchiment d'argent, moyennant la révision de la définition de l'infraction principale prévue par l'art. 305bis CPS.

2. Elargissement de la catégorie des infractions en relation avec la punissabilité de la participation à une organisation criminelle, moyennant la révision de l'art. 260ter CPS.

Deuxième catégorie: mesures contre l'escroquerie en matière fiscale ${ }^{t}$

3. Ajouter l'escroquerie en matière fiscale

3.1. parmi les infractions principales en amont du blanchiment d'argent d'après l'art. 305bis CPS;

3.2 parmi les infractions en relation avec la punissabilité de la participation à une organisation criminelle d'après l'art. 260ter CPS:

3.3. parmi les infractions dont le produit fait objet de confiscation d'après l'art. 59 CPS.

4. Extradition de personnes pour le chef d'escroquerie en matière fiscale moyennant la ratification du Protocole additionnel $n^{0} 99$ de la Convention européenne sur l'entraide internationale en matière pénale.

5. Remise à l'étranger du produit de l'escroquerie en matière fiscale.

Troisième catégorie: mesures contre la soustraction fiscale ${ }^{2}$

6. Ajouter la soustraction fiscale:

6.1. parmi les infractions principales en amont du blanchiment d'argent d'après l'art. 305bis CPS;

6.2. parmi les infractions en relation avec la punissabilité de la participation à une organisation criminelle d'après l'art. 260ter CPS;

6.3 parmi les infractions dont le produit fait l'objet de confiscation d'après l'art. 59 CPS.

7. Introduction de l'impôt à la source pour les avoirs de contribuables de l'Union européenne (projet Monti).

8. Gel et démantèlement des régimes fiscaux privilégiés pour des contribuables non-résidents en Suisse (d'après le modèle du Code de conduite adopté par l'UE - Ecofin - le 1 ${ }^{\text {er }}$ décembre 1997).

9. Criminalisation de la soustraction fiscale: si la punissabilité de la soustraction fiscale devait être élevée jusqu'au niveau de la peine privative de liberté,

9.1. la compétence de la poursuite étant attribuée à l'autorité judiciaire, le secret bancaire pourrait être levé;

9.2. pourrait être concédée l'extradition d'une personne recherchée à l'étranger pour soustraction fiscale;

9.3. pourrait être concédée la remise à l'étranger du produit de la soustraction fiscale.

10. Ratification de la Convention de coopération mutuelle entre les autorités fiscales du 25 juin 1987 de I'OCDE et du Conseil de l'Europe.

1. Par souci de cohérence interne avec l'art. 3 al. 3 EIMP, on utilise la définition d'escroquerie en matière fiscale prévue par l'art. 14 LDPA (Loi fédérale sur le droit pénal administratif).

2. Pour éviter toute confusion due à la diversité des définitions par le droit fiscal étranger, on utilise ici les définitions reconnues par le droit fiscal suisse. 


\section{ANNEXE 3: Nouvelles normes suisses contre la corruption Conséquences de la révision du Code pénal suisse du 22 décembre 1999}

1. Introduction de la punissabilité de la corruption active' de fonctionnaires étrangers

Celui qui a corrompu un fonctionnaire étranger ${ }^{2}$ est punissable aux mêmes conditions et avec la même peine qui sont prévues pour la punissabilité des fonctionnaires suisses, c'est-à-dire même

a) Iorsque les avantages offerts, promis et/ou assurés sont de caractère immatériel et indirect et non seulement lorsqu'ils sont de caractère matériel et direct;

b) Iorsque les destinataires des avantages sont des tierces personnes, c'est-à-dire des fiduciaires, des hommes de paille, des membres de la famille de la personne corrompue ou des partis politiques;

c) lorsque l'offre, la promesse et/ou l'assurance d'un avantage n'est pas destinée à ou n'est pas envisagée pour influencer le fonctionnaire, et cela même dans le cas de décisions d'appréciation ${ }^{3}$ ou dans le cas de facilitations dans le temps

d) Iorsque l'offre, la promesse et/ou la concession d'un avantage a lieu après la décision ou la mesure adoptée par l'administration

e) lorsqu'il n'y a aucun rapport entre l'avantage et un comportement concret du fonctionnaire, y compris les paiements de goodwill, le "pantouflage", les paiements pour maintenir une bonne atmosphère, etc.;

f) sans limitations aux paiements illicites dans le commerce international ${ }^{6}$.

2. Confiscation et saisie du produit de la corruption d'un fonctionnaire étranger, comme par exemple

a) les avantages qui ont été concédés aux fonctionnaires et/ou à une tierce personne:

b) les profits en faveur de la personne physique ou morale corruptrice provenant de la corruption, comme par exemple lors de la corruption dans le cadre d'appel d'offres, de la concession d'une autorisation, etc., qui ont eu lieu à cause de la corruption.

3. Punissabilité du blanchiment du produit de la corruption d'un fonctionnaire étranger, même dans le cas où le comportement corrupteur n'a aucune relation avec le territoire suisse, à condition qu'au moins une partie du produit ait été blanchie sur le territoire suisse.

4. Obligation de communication (d'après I'art. 9 LBA) et droit de communication (d'après l'art. 305ter chiffre 2 al. 2 CPS) concernant le produit de la corruption de fonctionnaires étrangers, lesquels au moins partiellement se trouvent dans la disponibilité d'un intermédiaire financier en Suisse.

5. Obligation de diligence accrue pour les banques suisses et leurs filiales à l'étranger concernant les relations d'affaires avec des personnalités étrangères qui exercent des fonctions politiques importantes (conformément aux Directives antiblanchiment N. 98/1 de la Commission fédérale des banques du 26 mars 1998, chiffres 4, 9, 15, 29 et 30).

6. Coopération internationale dans l'intérêt de poursuites pénales pour le chef de corruption de fonctionnaires étrangers, même si ces poursuites sont conduites par des Etats tiers, autres que l'Etat auquel appartient le fonctionnaire?

7. Interdiction de déduire des impôts les paiements effectués dans un but de corruption de fonctionnaires étrangers ${ }^{8}$.

1. La punissabilité de la corruption passive (soit le fonctionnaire qui se laisse corrompre) demeure réservée à l'Etat auquel appartient le fonctionnaire corrompu, même dans le cas où l'autorité de cet Etat ne devrait prendre aucune initiative. Néanmoins, les Etats tiers sont habilités à ne pas rester passifs, l'introduction de la punissabilité de la corruption de fonctionnaires étrangers ayant créé leur compétence pour la confiscation autonome du produit de la corruption, pour la poursuite d'office du blanchiment du produit de la corruption ainsi que de l'omission de communiquer les cas suspects, de même que pour l'adoption de mesures spéciales, comme lors de la saisie des avoirs appartenant à Marcos, Mobutu, Milosevic, Abacha ainsi qu'à leurs familles.

2. C'est-à-dire le membre d'une autorité politique ou le fonctionnaire d'un Etat étranger ou d'une organisation internationale.

3. Cf. R. Kaiser, Die Bestechung von schweizerischen Beamten unter Berücksichtigung des Vorentwurfs zur Revision des Korruptions des Strafrechts, Zürich, 1999, pp. 215 et suiv.

4. Cf. R. Kaiser, op. cit., pp. 238 et suiv.

5. En effet, le législateur suisse a renoncé à la condition de la "futuribilité" (cf. R. Kaiser, op. cit., p. 246).

6. En effet, cette limitation a été prévue par la Convention contre la corruption de I'OCDE du 1" décembre 1997 mais non par la Convention pénale contre la corruption du Conseil de l'Europe du 4 novembre 1998, laquelle sera aussi prochainement ratifiée par la Suisse.

7. Avant la révision du Code pénal, la condition de la double punissabilité n'était satisfaite que dans l'intérêt de poursuites pénales ouvertes dans l'Etat auquel appartient le fonctionnaire corrompu.

8. Dans ce but ont été révisées les lois fiscales fédérales et cantonales (art. 27, al. 3 et 59, al. 2 LIFD, et art. 10 al. 1 bis et 25, al 1 bis LHID). 


\section{Annexe 4}

\section{Matériaux concernant la révision du droit pénal contre la corruption}

\section{Organisations internationales}

a) Convention de I'OCDE pour la lutte contre la corruption de fonctionnaires étrangers dans le commerce international du $1^{\text {er }}$ décembre 1997.

b) Convention pénale contre la corruption du Conseil de l'Europe du 4 novembre 1998.

c) Convention civile contre la corruption du Conseil de l'Europe du 4 novembre 1999.

d) 20 Principes directeurs du Conseil de l'Europe pour la lutte contre la corruption, Résolution (97) 24 du 6 novembre 1997

e) Convention de I'Union européenne concernant la protection des intérêts financiers de la communauté du 26 juillet 1995, avec Protocole additionnel du 27 septembre 1996.

f) Convention de I'Union européenne concernant la lutte contre la corruption des fonctionnaires de I'Union européenne et des fonctionnaires des Etats membres du 26 mai 1997.

g) Convention interaméricaine contre la corruption du 29 mars 1996.

\section{Droit suisse}

a) Rapport final du groupe de travail "Contrôles de sécurité et corruption», Département fédéral de justice et police, Berne, octobre 1996.

b) Contrôle administratif du Conseil fédéral, danger de corruption et mesures de sécurité dans l'administration fédérale, rapport au Conseil fédéral du 26 mars 1998.

c) Message du Conseil fédéral no 99.026 du 19 avril 1999 concernant la révision du droit suisse contre la corruption, nouveaux articles 322 bis à 322 septies du Code pénal suisse, approuvé par le

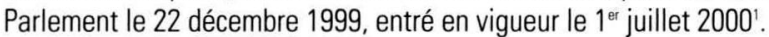

1. Eigen/Pieth (Hrsg.), Korruption im internationalen Geschäftsverkehr, Basel, 1998; R. Kaiser, Die Bestechung von schweizerischen Beamten unter Berücksichtigung des Vorentwurfs zur Revision des Korruptions des Strafrechts, Zürich, 1999; Transparency International, Berlin, site Internet www.transparency.de. 


\title{
AnNeXe 5 \\ Extrait de la Circulaire de la Commission fédérale des banques: Directives relatives à la prévention et à la lutte contre le blanchiment de capitaux (Blanchiment de capitaux) du 26 mars 1998
}

\author{
Chiffre marginal $n^{\circ} 4$ \\ «Parallèlement aux objectifs décris ci-dessus, ces directives visent également à formaliser quelques \\ principes précédemment dégagés par la Commission des banques en matière d'avoirs de personnes \\ exerçant des fonctions publiques importantes', notamment l'interdiction d'accepter des fonds provenant \\ de la corruption ou du détournement de biens publics, I'attention particulière à apporter à certaines \\ relations d'affaires et leur traitement au niveau de la direction. "

\section{Chiffre marginal $n^{\circ} 9$} \\ "Les intermédiaires financiers ne doivent pas accepter des fonds dont ils savent ou doivent présumer \\ qu'ils proviennent de la corruption ou de détournements de fonds publics. Ils doivent dès lors examiner \\ avec une attention particulière s'ils veulent entrer en relations d'affaires, accepter et garder des avoirs \\ appartenant, directement ou indirectement, à des personnes exerçant des fonctions publiques impor- \\ tantes pour un Etat étranger ou à des personnes et sociétés qui, de manière reconnaissable, leur sont \\ proches."
}

\section{Chiffre marginal $\mathrm{n}^{\circ} 15$}

\section{«4.1. Directives internes de l'intermédiaire financier}

Les intermédiaires financiers doivent édicter les instructions internes nécessaires à la prévention et à la lutte contre le blanchiment de capitaux en tenant compte des présentes directives. Ces instructions doivent prévoir notamment:

a) les cas dans lesquels le service spécialisé, au sens du chiffre 4.3., doit être impliqué:

b) la politique de relations d'affaires avec des personnes exerçant des fonctions publiques importantes pour un Etat étranger ou avec des personnes et sociétés qui, de manière reconnaissable, leur sont proches:

c) I'attribution exclusive à la direction, ou à des membres de celle-ci, des compétences relatives à I'entrée en relation d'affaires avec les personnes mentionnées à la lettre b) et le devoir de la direction d'examiner régulièrement de telles relations d'affaires." "

\section{Chiffre marginal $n^{\circ} 29$}

"Lorsqu'un intermédiaire financier en cas de doute portant sur l'existence d'un cas de blanchiment, mais en l'absence de soupçons fondés, rompt les relations d'affaires sans informer les autorités compétentes, il ne doit admettre le retrait des avoirs que sous une forme qui permette, au besoin, aux autorités de poursuite pénale d'en retrouver la trace ("paper trail"). II s'abstiendra de procéder à des paiements comptants ou à des livraisons physiques de titres et de métaux précieux dans une mesure significative. Ces obligations valent également si l'intermédiaire financier a un doute portant sur l'existence d'un cas de corruption ou de détournement de biens publics."

\section{Chiffre marginal $n^{0} 30$}

"Dans les cas mentionnés au § 29, l'intermédiaire financier ne peut rompre les relations d'affaires ou autoriser le retrait de montants importants s'il existe des indices concrets que des mesures de sûreté d'une autorité sont imminentes."

1. Ce domaine se rapproche de celui du blanchiment dans la mesure où l'intermédiaire financier doit exercer sa vigilance et respecter certaines exigences de manière similaire. En outre, certains comportements illicites de politiques ou de fonctionnaires sont saisis sous l'angle du blanchiment, s'il s'agit de crimes. Sont également susceptibles de motiver l'application de cette norme dans un proche avenir d'autres comportements, telle la corruption de fonctionnaires étrangers, de sorte qu'il se justifie d'introduire ces principes dans la présente circulaire. 